\title{
Estudio de los caballos del yacimiento de Quibas, Pleistoceno Inferior final (Abanilla, Murcia, España)
}

\section{Study of horses from the Quibas site, latest Early Pleistocene (Abanilla, Murcia, Spain)}

\author{
P. Piñero', M.T. Alberdi \\ 1 Institut Català de Paleoecologia Humana i Evolució Social (IPHES), Zona Educacional 4-Campus Sescelades URV \\ (Edifici W3), 43007, Tarragona, España. Email: ppinero@iphes.cat \\ 2 Departamento de Paleobiología, Museo Nacional de Ciencias Naturales, CSIC, José Gutiérrez Abascal 2, 28006, Madrid, \\ España. Email: alberdi.maite@gmail.com
}

\section{RESUMEN}

En este artículo se describen más de 60 restos fósiles de équido recuperados en el yacimiento kárstico del Pleistoceno Inferior final de Quibas (Abanilla, Murcia). Se hace un estudio de su taxonomía y morfología, estado evolutivo y los factores paleoclimáticos vinculados. Comparando con fósiles de équidos hallados en otras localidades españolas y europeas a través de análisis multivariantes, los restos se asignan en su mayor parte a la especie de tamaño medio Equus altidens, último eslabón del linaje de los caballos estenonianos. En España, esta especie se relaciona con unas condiciones climáticas cálidas o cálidas-templadas, cuya vegetación principal estaría compuesta de bosque o bosque-sabana. Se constata también una escasa presencia de Equus suessenbornensis por primera vez en el yacimiento de Quibas.

Palabras clave: Pleistoceno Inferior final; Quibas; Equus altidens; estenoniano; Equus suessenbornensis.

\section{ABSTRACT}

In this paper, over 60 fossil horse remains from the latest early Pleistocene karstic site of Quibas (Abanilla, Murcia, Spain) are described. Their taxonomy and morphology, evolutionary stage, and paleoecological determining factors are studied. The multivariate analysis through comparison with different forms that occur in other Spanish and European localities shows that the majority of the fossils match the medium-sized species Equus altidens. This species represents the last form in the stenonoid lineage. In Spain, $E$. altidens is related to warm or warm-temperate climate, in woodlands or savanna-mosaic habitats. Equus suessenbornensis is also sparsely represented, first cited in the site of Quibas.

Keywords: Early latest Pleistocene; Quibas; Equus altidens; stenonoid; Equus suessenbornensis. 


\section{Introducción}

El yacimiento paleontológico de Quibas (Abanilla, Murcia) está situado en la ladera sureste de la Sierra de Quibas, en el paraje denominado Collado del Rey, al pie de una cantera abandonada a una altitud de $669 \mathrm{msnm}$ (Fig. 1). Ofrece un registro fósil tanto de vertebrados como de invertebrados del Pleistoceno Inferior y se identifican más de 60 especies (Alba et al., 2011; Blain et al., 2014; Carlos-Calero et al., 2004, 2006a, b; Van der Made et al., 2007; Montoya et al., 1999, 2001). Se establece para este yacimiento kárstico una edad aproximada entre 1,4 y 1,2 Ma (Piñero et al., 2015). Los caballos de este yacimiento han sido estudiados de forma preliminar por Montoya et al. $(1999,2001)$ identificando la especie Equus altidens a partir de un escaso número de dientes bastante desgastados. Sin embargo, la aparición en campañas posteriores de nuevos y abundantes restos de équidos (desde 2002 a 2009), permiten llevar a cabo un estudio morfológico y taxonómico más profundo, y comparar con otros restos de équidos hallados en otras localidades españolas y europeas.

El género Equus está ampliamente distribuido en España, cuya presencia se constata desde el Villafranquiense medio hasta el Holoceno. Se pueden diferenciar dos grupos de caballos en Europa durante este intervalo temporal: "estenonoides" (linguafléxido en forma de V) y "caballoides" (linguafléxido en forma de U). Los estenonoides entran en Eurasia al menos durante el Villafranquiense medio, siendo los primeros caballos monodáctilos que llegan al continente (Lindsay et al., 1980; Azzaroli, 1983). Se trata de elementos comunes entre el Plioceno Superior y el Pleistoceno Inferior-Medio. A comienzos del Pleistoceno Medio son reemplazados por los caballoides, favorecidos posiblemente por los cambios climáticos (Alberdi et al., 1998). Entre los caballos estenonoides se encuentra la especie Equus altidens, la cual se considera como el último eslabón del linaje de los caballos estenonianos (Alberdi et al., 1998). Se presentan dos subespecies en España, que aunque muy próximas entre sí, muestran diferencias de talla y gracilidad. Equus altidens altidens (subespecie tipo) desarrolla un menor tamaño y una mayor gracilidad respecto a la segunda subespecie Equus altidens granatensis. La primera se ha descrito en Süssenborn (localidad tipo), Solhilac, Venosa-Loreto, Pirro Nord, Huéscar-1, Cúllar de Baza-1 y Cueva Victoria, entre otras localidades. La segunda en Láchar, Fuensanta, Venta Micena, Fuente Nueva-3 y Barranco León-5 (Alberdi \& Ruiz Bustos, 1989; Alberdi et al., 1998; Alberdi, 2010; Alberdi \& Palombo 2013a, b; Alberdi \& Piñero, 2015). Equus suessenbornensis es un caballo de grandes dimensiones que presenta caracteres estenonianos y caballinos combinados. Cohabita con Equus altidens en numerosas localidades españolas como Huéscar-1, Cúllar de Baza-1, Fuente Nueva-3 y Barranco León-5 (Alberdi et al., 1998). Esta asociación también aparece en localidades italianas como Venosa-Loreto y Pirro Nord (Alberdi et al., 1988; Alberdi \& Palombo, 2013a, b), en Süssenborn, Alemania, localidad tipo de ambas especies (Musil, 1969), y en Solilhac, Francia (Prat 1980).

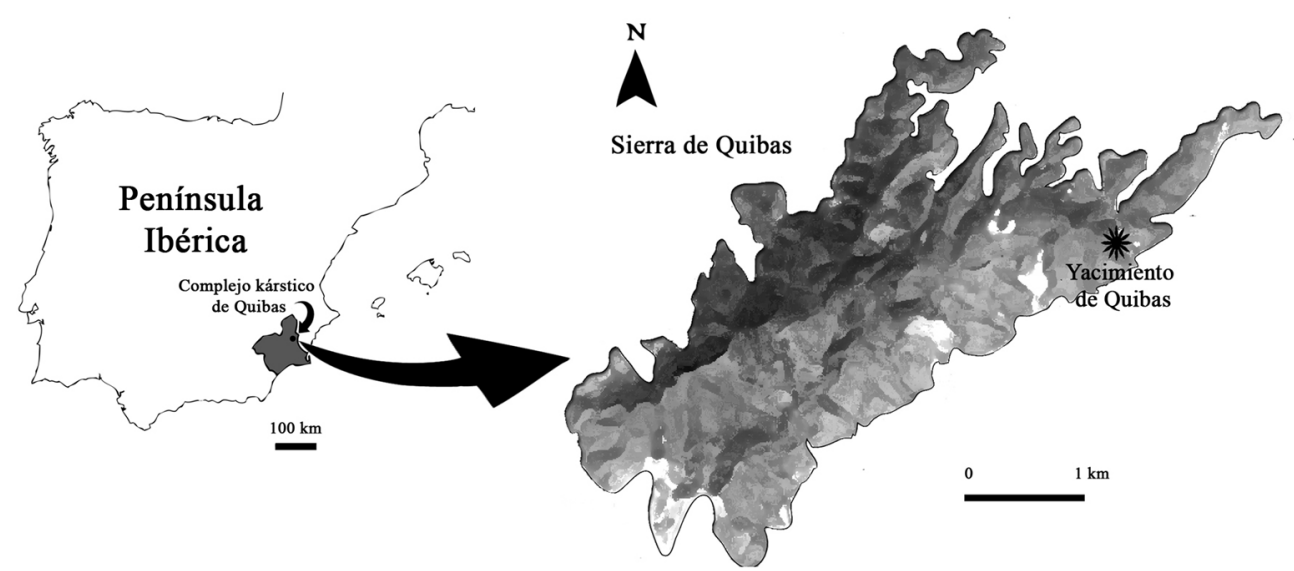

Fig. 1.—Localización geográfica del yacimiento de Quibas. 


\section{Marco Geológico}

La Sierra de Quibas representa un macizo carbonatado de $6 \mathrm{~km}$ de longitud y 2,5 $\mathrm{km}$ de anchura, de dirección NE-SE. Localizado en la Zona Subbética Media, está compuesto principalmente por calizas y dolomías Jurásicas (Rodríguez-Estrella et al., 2004). La afección de una gran actividad kárstica durante el Plio-Pleistoceno, condujo a un importante número de estructuras exokársticas y endokársticas, algunas de ellas totalmente colmatadas en la actualidad por rellenos de carácter mixto detrítico y de precipitación química. En una de estas estructuras se localiza el yacimiento paleontológico objeto de estudio (Durán et al., 2004), constituyendo así un relleno fosilífero del Pleistoceno Inferior que colmata cavidades situadas en el seno de dolomías del Lías inferior (Jurásico).

La mayor parte de los fósiles provienen de una galería de $5 \mathrm{~m}$ de anchura y $9 \mathrm{~m}$ de altura (conocida como "Entrada Cueva" $=\mathrm{EC}$ en los mapas del sistema kárstico) que se extiende más de $30 \mathrm{~m}$ longitudinalmente hacia el interior de la sierra, presentando brechas laterales separadas por espeleotemas cálcicos ( 2 en fig. 2). También se puede identificar una sima de $20 \mathrm{~m}$ de profundidad y $2 \mathrm{~m}$ de anchura (1 en fig. 2). Aunque ambas estructuras aparecen separadas por un espeleotema cálcico de $3 \mathrm{~m}$ de espesor, probablemente se muestren conectadas en su interior (Fumanal \& Bláquez en Montoya et al., 1999). Una tercera cavidad colmatada conocida como Gruta 1 (3 en fig. 2) ha sido reconocida en trabajos posteriores (Cuadros, 2010; Piñero, 2012; Piñero et al., 2015). En la parte basal del yacimiento se pudieron distinguir seis capas, compuestas principalmente por arcillas, limos y brechas más o menos cementadas, aunque actualmente esta sección se encuentra cubierta por derrubios (Fig. 2).

\section{Material y Método}

Los restos fósiles estudiados en este trabajo proceden del yacimiento de Quibas y se encuentran depositados en el Museo de Arqueología de Murcia (MAM). Aunque se han recuperado más de 80 restos de équido, en el estudio se excluyen todos aquellos que no aportan información taxonómica, tales como fragmentos indeterminados, vértebras, costillas, incisivos o caninos. Dentición superior: 8 dientes aislados consistentes en 1 D1, 3 P3-4 derechos, 4 M1-2 (3 derechos y 1 izquierdo); dientes inferiores: serie inferior p2-m3 izquierda deformada, y 2 m1-2 izquierdos aislados. Esqueleto postcraneal: 1 radio completo, 2 epífisis proximales y 1 distal; 6 McIII completos,

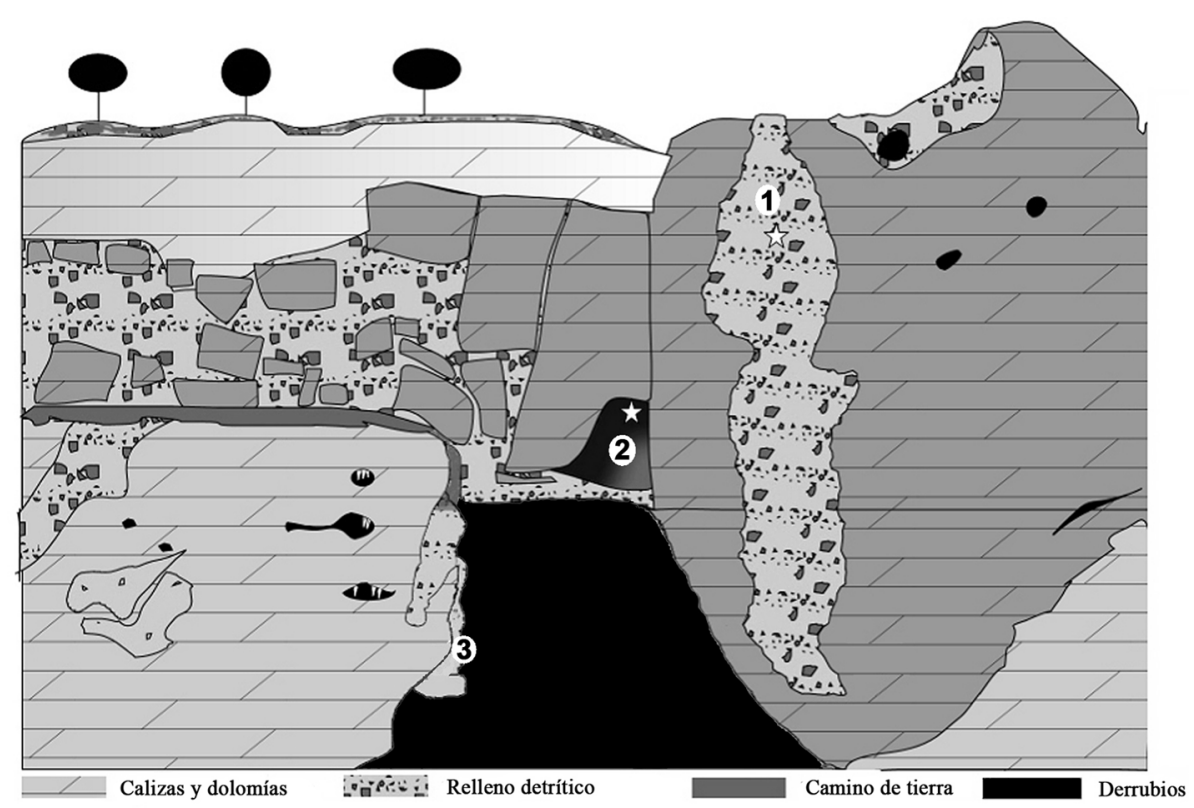

Fig. 2._Perfil del yacimiento de Quibas. 1, Sima; 2, Entrada Cueva; 3, Gruta 1. Tomado de Cuadros, 2010. 
2 juveniles; 3 fragmentos de metápodos laterales; 2 fragmentos de pelvis; 1 fémur completo, 2 fragmentos: 1 cóndilo y 1 extremo distal; 1 tibia completa, 2 extremos proximales, 1 diáfasis y 1 extremo distal (unido al astrágalo); 2 calcáneos enteros; 2 astrágalos completos; 2 MtIII completos; 5 1FIII completas y 2 epífisis distales; 6 2FIII completas; y 5 3FIII, 4 completas (abreviaturas en Eisenmann et al., 1988).

La descripción morfológica de los molares se ha realizado siguiendo las recomendaciones de la "Hipparion Conference" (Eisenmann et al., 1988). Se han calculado los índices de gracilidad en McIII y MtIII con el objeto de compararlos con otras localidades. Estos índices se han calculado contrastando las dimensiones $1 \mathrm{y}$ 3 y 1 y 11: IG-1: McIII3-MtIII3×100/McIII1-MtIII1, donde McIII3/MtIII3 es el diámetro máximo transverso en la diáfisis de metacarpianos y metatarsianos y McIII1/MtIII1 es su longitud máxima; IG-2: McIII11-MtIII11×100/McIII1-MtIII1, donde McIII11/MtIII11 representa el diámetro transversal máximo de la extremidad distal de metacarpianos y metatarsianos, respectivamente (Bernor et al., 1990). También se ha realizado una estimación del peso del caballo siguiendo a Alberdi et al. (1995), a partir de la profundidad mínima distal del cóndilo medial en el McIII (McIII13), y de la profundidad proximal de la 1FIII (F5).

Se han realizado análisis bivariantes y multivariantes, utilizando los parámetros recomendados por la "Hipparion Conference" (Eisenmann et al., 1988). Con las dimensiones de los dientes yugales superiores e inferiores se han llevado a cabo análisis bivariantes contrastando longitud mesio-distal y anchura buco-lingual de cada diente a $1 \mathrm{~cm}$ de la base con el fin de que la dispersión sea menor (sólo se han incluido los dientes procedentes de Quibas). Con las dimensiones de los restos óseos del esqueleto apendicular (MCIII, AST, CAL, MTIII, 1FIII y 2FIII) se han realizado análisis multivariantes, en especial análisis discriminantes (AD). Estos análisis discriminantes se han llevado a cabo a través del método de incluir todas las variables juntas dejando fuera las de la localidad en estudio con el fin de que sus restos se agrupen con los restos de aquellos ejemplares más próximos. Para ello, se han usado las bases de datos de uno de los autores (MTA) correspondientes a los restos fósiles de los équidos de final del Pleistoceno Inferior e inicio del Pleistoceno Medio ya estudiados y determinados taxonómicamente. Estos análisis se han realizado sobre matrices de $41 \mathrm{MCIII}, 46 \mathrm{AST}$, 8 CAL, 43 MTIII, 50 1FIII y 54 2FIII de Equus procedentes de las localidades de Cueva Victoria, Huéscar-1 y Venta Micena en España; VenosaLoreto y Ponte Galeria en Italia; Solilhac en Francia; y Süssenborn en Alemania (Alberdi, 2010; Alberdi \& Palombo, 2013a, b; Alberdi \& Piñero, 2015). Los análisis se han realizado mediante el paquete estadístico SPSS 15.0. Detalles sobre estos análisis se pueden encontrar en Reyment (1991). Todas las dimensiones se expresan en milímetros.

\section{Descripción}

Los restos fósiles del yacimiento de Quibas corresponderían a un caballo de talla media, excepto las piezas dentarias Q04-EcM-31 y Q07-Ect-34c que presentan un mayor tamaño. Son muy similares morfológicamente a otros especímenes de équidos del Pleistoceno Inferior final y principios del Pleistoceno Medio (ver tablas 1, 2, 3 y 4, y figuras 3, 4 y 5).

En los dientes superiores aislados (A en fig. 3), el protocono tiene un tamaño reducido, con una forma triangular en todos los ejemplares, excepto en Q07-Ect-34g (P3-4 derecho) que es ovalado, casi circular. Presenta entre 1 y 2 pliegues caballinos. El hipocono tiene forma ovalada en los M1-2 y, en el caso de Q07-Ect-34b (M1-2 derecho), desarrolla tres pliegues en su parte labial, mientras que es circular y abierto en los P3-4. Los pliegues de las fosetas no son numerosos, de forma que la parte anterior de la prefoseta alcanza un máximo de 2 pliegues, mientras que en su parte posterior se llegan a contar 3. La parte anterior de la postfoseta llega a alcanzar hasta 6 pliegues, mientras que en su parte distal el máximo está en 3. El parastilo y el mesostilo son fuertes en premolares y más estrechos en molares.

Entre los dientes inferiores se dispone de una única serie dental que, aunque completa, se presenta en tres piezas separadas, Q03-2-1(a), Q03-2-1(b) y Q03-2-1(c). Sus dimensiones son: L p2-m3=167,9, $\mathrm{L}$ p2-4=86,5 y L m1-m3=80,4. Estas dimensiones son muy similares a las dimensiones de las series procedentes de Cueva Victoria (Alberdi \& Piñero, 2015) y más pequeñas que las dadas por Marín (1987) para Venta Micena.

Los dientes inferiores aislados (A en fig. 3), presentan un lazo metacónido-metastílido con metacónido redondeado y metastílido más bien anguloso. 
Tabla 1.-Dimensiones de la dentición superior e inferior de Equus de la localidad de Quibas (Murcia). Abreviaturas: LS, longitud en la superficie oclusal; AS, anchura en la superficie oclusal; LB, longitud a $1 \mathrm{~cm}$ de la base; $A B$, anchura a $1 \mathrm{~cm}$ de la base; H, altura; L PR, longitud del protocono; PL C, número de pliegues caballinos; PLS FOS, número de pliegues en las fosetas;

L POSTFL, longitud del postfléxido; L LAZO, longitud del lazo

\begin{tabular}{|c|c|c|c|c|c|c|c|c|c|c|}
\hline \multicolumn{11}{|c|}{ DENTICIÓN SUPERIOR } \\
\hline Sigla & TIPO & LS & AS & LB & $\mathrm{AB}$ & $\mathrm{H}$ & LPR & PLC & PLS FOS & OBSERVACIONES \\
\hline Q07-Ect-34a & M1-2 izquierdo & 33,8 & 25 & 24,2 & 27,3 & 74 & 9,2 & 2 & $2 / 11 / 2$ & \\
\hline Q07-Ect-34c & M1-2 derecho & 32,5 & 24 & 27,8 & 27,1 & & & & & en germen \\
\hline Q07-Ect-34e & M1-2 derecho & 32,2 & 26,1 & & & & & & & en germen \\
\hline Q07-Ect-34f & P3-4 derecho & 33 & 25,9 & & & & 9,9 & 1 & $1 / 32 / 0$ & \\
\hline Q07-Ect-34g & P3-4 derecho & 31,5 & 26,9 & & & & & 1 & $0 / 06 / 0$ & \\
\hline Q07-Ect-34b & M1-2 derecho & 33 & 25,1 & 25 & 27,5 & 73,8 & 9,2 & 2 & $2 / 03 / 3$ & \\
\hline Q07-Ect-34d & P3-4 derecho & 35 & 31,3 & & & & & & & en germen \\
\hline QB-56 & $\mathrm{D} 2$ & 37 & 24,3 & & & 25,7 & & & & \\
\hline \multicolumn{11}{|c|}{ DENTICIÓN INFERIOR } \\
\hline Sigla & TIPO & LS & AS & LB & $A B$ & $\mathrm{H}$ & L POSTFL & L LAZO & & OBSERVACIONES \\
\hline Q04-EcM-31 & m1-2 izquierdo & 30,8 & 16,4 & 26,9 & 14,8 & & 11,3 & 18,1 & & \\
\hline Q03-E-20 & m1-2 izquierdo & 23,5 & 13,3 & 22 & 13,9 & 28,8 & 5,9 & 12,4 & & \\
\hline Q03-22-1(c) & m3 izquierdo & 31,1 & 13 & 31,9 & 11 & 38,2 & 6,9 & 14 & & \\
\hline Q03-22-1(b) & m2 izquierdo & 26,1 & 13,2 & 23,1 & 13 & 40 & 7,3 & 12,9 & & Fracturado \\
\hline Q03-22-1(a1) & m1 izquierdo & 23,2 & 14 & 23,5 & 11,2 & 38,7 & 7,3 & 13,1 & & \\
\hline Q03-22-1(a2) & p4 izquierdo & 29 & 16 & 25,6 & & 41,1 & 11 & 15,5 & & \\
\hline Q03-22-1(a3) & p3 izquierdo & 28,3 & 15,2 & & & & 12,5 & 17,8 & & \\
\hline Q03-22-1(a4) & p2 izquierdo & 29,2 & 14 & & & & 14,6 & 12 & & \\
\hline
\end{tabular}

Tabla 2.-Dimensiones de los huesos largos de Equus altidens del yacimiento de Quibas (Murcia). Los números corresponden a la nomenclatura recomendada en la "Hipparion Conference" (Eisenmann et al., 1988). ca=cálculo aproximado

\begin{tabular}{|c|c|c|c|c|c|c|c|c|c|c|c|c|c|}
\hline RADIO & & & & & & & & & & & & & \\
\hline Sigla & TIPO & 1 & 2 & 3 & 4 & 5 & 6 & 7 & 8 & 9 & 10 & 11 & 12 \\
\hline Q08-Ect1-10I & Epífisis distal izquierda & & & & & & & & 56,92 & 34,87 & 72,35 & 25,45 & 14,86 \\
\hline Q09-Ect1-2b & $\begin{array}{l}\text { Epífisis proximal } \\
\text { juvenil }\end{array}$ & & & 31,5 & 21,33 & & & & & & & & \\
\hline Q08-Ect1-3a & Epifisis proximal & & & & & 61,77 & 32,93 & & & & & & \\
\hline Q07-Ect-149 & Radio derecho & 356,3 & 342,2 & 37 & 26,87 & 70,61 & 38 & 72,26 & ca 60,7 & ca 32,33 & ca 71 & & \\
\hline Q09-Ect1-2j & Epífisis distal derecha & & & & & & & & ca 55,2 & 35 & 73,8 & 25,9 & 13,4 \\
\hline \multicolumn{14}{|l|}{ FÉMUR } \\
\hline Sigla & TIPO & 1 & 2 & 3 & 4 & 5 & 6 & 7 & 8 & 9 & 10 & & \\
\hline Q08-Ect1-10a & Fémur derecho & 381,6 & 34,5 & 36,16 & 43,83 & 111,4 & 83,12 & 87,1 & 106,28 & 57,4 & 48 & & \\
\hline Q09-Ect1-13 & Epífisis distal izquierdo & & & & & & & & & 54,7 & & & \\
\hline Q08-Ect1-10j & Cóndilo & & & & & & & & & & 50,55 & & \\
\hline
\end{tabular}

\begin{tabular}{|c|c|c|c|c|c|c|c|c|c|c|}
\hline \multicolumn{11}{|l|}{ TIBIA } \\
\hline Sigla & TIPO & 1 & 2 & 3 & 4 & 5 & 6 & 7 & 8 & 9 \\
\hline Q08-Ect1-10b & Tibia derecha & 354,4 & 337,28 & 40,86 & 33,45 & 89,12 & 83 & 64,1 & 44,2 & 50,35 \\
\hline Q09-Ect1-18 & $\begin{array}{l}\text { Epífisis proximal } \\
\text { derecha }\end{array}$ & & & & 86,77 & 65,5 & & & & \\
\hline Q09-Ect1-12b & $\begin{array}{l}\text { Epífisis proximal } \\
\text { izquierda }\end{array}$ & & & & ca 80,8 & ca 61,9 & & & & \\
\hline Q09-Ect1-20 & Diáfisis & & & 34,35 & 25,72 & & & & & \\
\hline Q09-7 & Epífisis distal derecha & & & & & & & 74,8 & ca 45,2 & \\
\hline
\end{tabular}




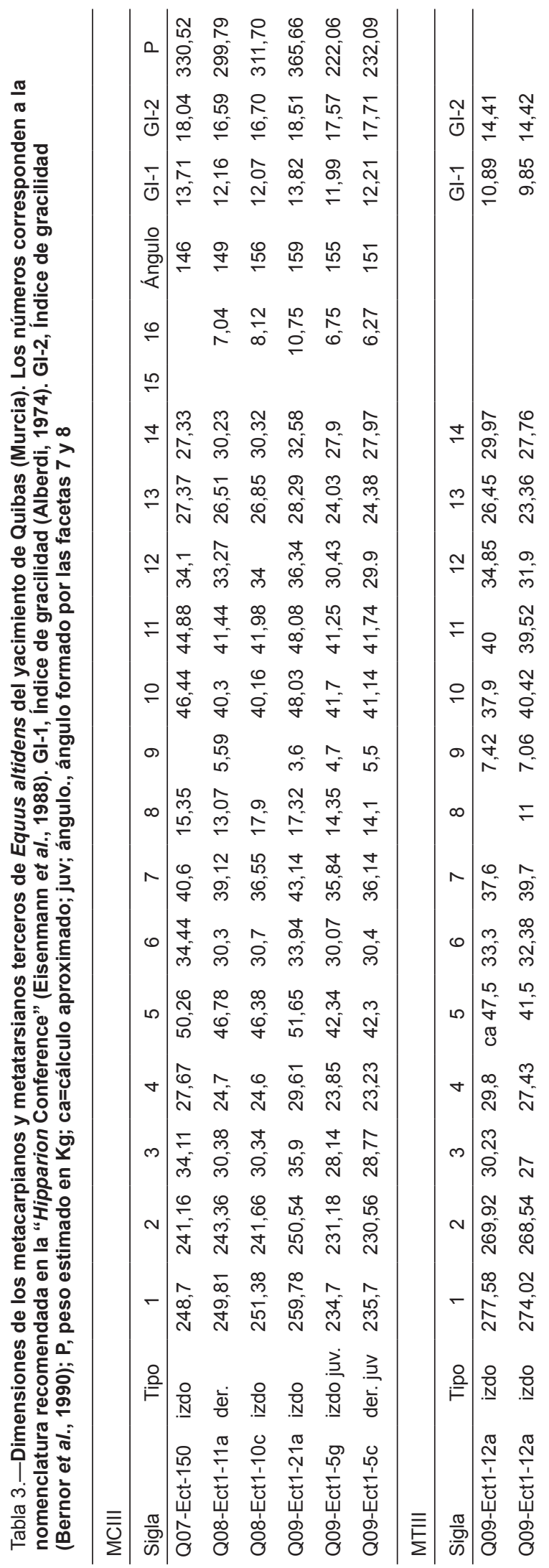

El ectofléxido en los premolares no llega a atravesar el istmo formado por el prefléxido y el postfléxido, mientras que en los molares penetra en el lazo, llegando a contactar con el linguafléxido. Ni el postfléxido ni el prefléxido muestran el esmalte plegado. El linguafléxido es generalmente anguloso, en forma de V. El dibujo oclusal va desapareciendo con el desgaste del diente. El m1-2 Q04-EcM-31, aunque de mayor tamaño que el resto de piezas, presenta un esquema morfológico similar.

Los restos del esqueleto apendicular no son muy abundantes (Figs. 4 y 5). De los 13 restos de huesos largos existentes, sólo tres están completos, una tibia y un fémur de un mismo individuo (Fig. 4, localizados en conexión anatómica) y un radio. Comparados con los huesos largos de la localidad de Pirro Nord (Alberdi \& Palombo, 2013a, b), entran dentro de su variabilidad de talla. Las longitudes del radio y la tibia son muy próximas a las de Venta Micena. Los 8 metápodos: 6 metacarpianos (fig. 3 B) y 2 metatarsianos (Fig. 4: 4 y 5) son de tamaño medio. Sus índices de gracilidad son: IG-1 en McIII varía entre 12,07 y 13,82 y en MtIII entre 9,85 y 10,89 ; IG-2 en McIII varía entre 16,59 y 18,51 y en MtIII entre 14,41 y 14,42 . Estos índices indican que los metapodiales de Quibas son más gráciles que los de Pirro Nord, pero entran en la distribución de los de Venta Micena.

La estimación del peso (tablas 3 y 4), siguiendo a Alberdi et al. (1995), ofrece valores más reducidos que los estimados para E. altidens granatensis y más próximos a $E$. altidens altidens.

\section{Resultados}

\section{Análisis morfológicos}

Los restos estudiados presentan caracteres morfológicos típicos de la especie Equus altidens. La descripción realizada en el apartado anterior confirma que la morfología de los dientes presenta caracteres estenonianos derivados. Esto es, la presencia de un protocono con forma subtriangular y con una pequeña hendidura lingual, cuya parte distal es un poco más alargada que en la especie E. stenonis. En los dientes inferiores, el metacónido redondeadoovalado y el metastílido con una forma más angulosa que en E. stenonis, forman el doble nudo o lazo diferente. 
Tabla 4.-Dimensiones de las falanges de Equus altidens del yacimiento de Quibas (Murcia). Los números corresponden a la nomenclatura recomendada en la "Hipparion Conference" (Eisenmann et al., 1988). Abreviaturas como en Tabla 3

\begin{tabular}{|c|c|c|c|c|c|c|c|c|c|c|}
\hline \multicolumn{11}{|l|}{$1 \mathrm{FIII}$} \\
\hline Sigla & 1 & 2 & 3 & 4 & 5 & 6 & 7 & 8 & 9 & $P$ \\
\hline Q09-Ect1-5d & 81,85 & 74,25 & 25,4 & 40,7 & 30,76 & 34,78 & 36,56 & 20,3 & 47 & 213,05 \\
\hline Q09-Ect1-21b & 90,9 & 85,3 & 33,66 & 48,15 & 34,7 & 40,48 & 42,37 & 25,7 & 51,9 & 323,91 \\
\hline Q09-Ect1-2i & 76,7 & 70,58 & 24,03 & 41,7 & 32,47 & 33,11 & 33,6 & 19,6 & 43,03 & 257,13 \\
\hline Q08-Ect1-10d & 80,47 & 72,6 & 25,5 & 40,68 & 32,48 & 33,76 & 34,7 & 22,3 & 42,67 & 257,40 \\
\hline Q08-Ect1-11b & 87 & 80,2 & 26,68 & 40,38 & 33,66 & 36,16 & 37,48 & 22,88 & 50,74 & 291,40 \\
\hline Q05-Ei-77 juv. & & & 25 & & & 33,5 & 32,8 & 20 & & \\
\hline Q05-Ei-76 juv. & & & & & & & 31,4 & 21,2 & & \\
\hline \multicolumn{11}{|l|}{$2 \mathrm{FIII}$} \\
\hline Sigla & 1 & 2 & 3 & 4 & 5 & 6 & 7 & 8 & 9 & \\
\hline Q09-Ect1-10c & 44,15 & 33,1 & 34 & 39,67 & 29,55 & 36,7 & & & & \\
\hline Q09-Ect1-5e & 41,75 & 30,1 & 35,9 & 40,2 & 26,67 & 39,7 & & & & \\
\hline Q09-Ect1-3 & 43,75 & 33,65 & 35,7 & 41,18 & 29,14 & 40,8 & & & & \\
\hline Q09-Ect1-10e & 44,15 & 32,88 & 34,21 & 39,8 & 28,64 & 36,92 & & & & \\
\hline Q08-Ect1-10g & 43,7 & 32,8 & 36,1 & 41,2 & 29,8 & 41,4 & & & & \\
\hline Q09-Ect1-3c & 44,4 & 33,9 & 39 & 45,8 & 30,9 & 42,1 & & & & \\
\hline \multicolumn{11}{|l|}{$3 F I I I$} \\
\hline Sigla & 1 & 2 & 3 & 4 & 5 & 6 & 7 & 8 & 9 & \\
\hline Q09-Ect1-5f & 42,16 & 41,22 & 43,28 & 40,87 & 25,7 & 32,32 & 36 & 116,7 & & \\
\hline Q09-Ect1-5h & 41,97 & 41,54 & 44 & 39,8 & 24,71 & 30,6 & 36 & 114,05 & & \\
\hline Q09-Ect1-10b & 46,73 & 46 & 44 & 37,45 & 25,93 & 33,66 & 32 & 119,4 & & \\
\hline Q09-Ect1-10a & 47,32 & 43,5 & 51,8 & 39 & 27,12 & 35,62 & 31 & 118,3 & & \\
\hline Q08-Ect1-10i & 46 & 45,1 & ca 45 & 36,23 & 27,3 & 29,3 & 33 & & & \\
\hline
\end{tabular}

Por otra parte, los huesos del esqueleto apendicular, en especial los metápodos y falanges, son más gráciles que los estenonoides típicos (localidades de Olivola, Matassinos y Valdarno superior en Italia, Alberdi et al., 1998). En cuanto al peso, según las dimensiones de MCIII13, ofrece un promedio de $326 \mathrm{Kg}$, mientras que según las dimensiones de 1FIII5 la media es de $268 \mathrm{Kg}$. En cualquier caso, son menos pesados que los estenonianos típicos (peso medio a partir de 1FIII5 de $408 \mathrm{Kg}$, Alberdi et al., 1995).

\section{Análisis estadísticos}

Los análisis bivariantes realizados con los dientes yugales contrastando la longitud y la anchura de cada diente a $1 \mathrm{~cm}$ de la base (Fig. 6), con el fin de que la dispersión sea menor, indican que de todas maneras la dispersión es muy alta tanto en dientes superiores como en inferiores. Por ello, estos análisis no ayudan a dilucidar la identificación taxonómica subespecífica de los restos, ya que tanto los restos de E. a. altidens como los de E. a. granatensis tienen una amplia dispersión. No obstante, sí se observa que dos de las piezas dentarias (Q04-EcM-31 y Q07-Ect-34c) se aproximan mucho a Equus suessenbornesis de Cueva Victoria, Fuente Nueva 3 y Barranco León 5, permitiendo identificar la presencia de dicha especie en el yacimiento de Quibas (ver fig. 6), ya que la morfología dentaria de ambas especies es muy similar.

Los resultados de los análisis discriminantes (Fig. 7; Tabla 5) permiten observar con cuáles de las poblaciones ya estudiadas se agrupan los restos óseos de Quibas, así como el grado de fiabilidad de los resultados obtenidos.

Los resultados del análisis discriminante para los McIII indican que el 91,1\% de los casos agrupados originalmente estaban correctamente clasificados, mientras que aplicando la validación cruzada la clasificación previa correcta alcanza el 89,3\%. Los restos de Quibas se agrupan tres con E. a. granatensis 
Tabla 5.-Porcentaje de clasificación correcta obtenido de los análisis discriminantes por validación cruzada. 1, E. a. altidens; 2, E. suessenbornensis; 3, E. a. granatensis; desagrupados, los ejemplares de Quibas

\begin{tabular}{|c|c|c|c|c|c|}
\hline & Original & $\mathrm{N}$ & 1 & 2 & 3 \\
\hline \multirow[t]{4}{*}{ McIII } & 1 & 20 & $85 \%(17)$ & 0 & $15 \%(3)$ \\
\hline & 2 & 11 & 0 & $81,8 \%(9)$ & $18,2 \%(2)$ \\
\hline & 3 & 25 & 0 & 0 & $100 \%(25)$ \\
\hline & Desagrupados & 4 & $25 \%(1)$ & 0 & $75 \%(3)$ \\
\hline \multirow[t]{4}{*}{ MtIII } & 1 & 23 & $78,3 \%(18)$ & $4,3 \%(2)$ & $17,4 \%(4)$ \\
\hline & 2 & 18 & $11,1 \%(2)$ & $83,3 \%(15)$ & $5,6 \%(1)$ \\
\hline & 3 & 30 & $6,7 \%(2)$ & $16,7 \%(5)$ & $76,7 \%(23)$ \\
\hline & Desagrupados & 2 & $50 \%(1)$ & 0 & $50 \%(1)$ \\
\hline \multirow[t]{4}{*}{ Astragalus } & 1 & 27 & $77,8 \%(21)$ & $3,7 \%(1)$ & $18,5 \%(5)$ \\
\hline & 2 & 15 & $6,7 \%(1)$ & $93,3 \%(14)$ & 0 \\
\hline & 3 & 7 & $28,6 \%(2)$ & 0 & $71,4 \%(5)$ \\
\hline & Desagrupados & 2 & $100 \%(2)$ & 0 & 0 \\
\hline \multirow[t]{4}{*}{ 1FIII } & 1 & 35 & $91,4 \%(32)$ & 0 & $8,6 \%(3)$ \\
\hline & 2 & 14 & 0 & $100 \%(14)$ & 0 \\
\hline & 3 & 6 & $33,3 \%(2)$ & 0 & $66,7 \%(4)$ \\
\hline & Desagrupados & 5 & $100 \%(5)$ & 0 & 0 \\
\hline \multirow[t]{4}{*}{$2 \mathrm{FIII}$} & 1 & 29 & $75,9 \%(22)$ & 0 & $24,1 \%(7)$ \\
\hline & 2 & 22 & $13,6 \%(3)$ & $77,3 \%(17)$ & $9,1 \%(2)$ \\
\hline & 3 & 3 & 0 & 0 & $100 \%(3)$ \\
\hline & Desagrupados & 4 & $75 \%(3)$ & 0 & $25 \%(1)$ \\
\hline
\end{tabular}

y uno con E. a. altidens. En la figura 7 queda claro que la mayoría de los restos de Quibas se agrupan con los restos de E. a. granatensis. También se observa que E. a. granatensis está mejor acotado que E. a. altidens.

En el caso de los astrágalos, los resultados indican una clasificación previa correcta del $81,6 \%$ y del 77,6\% con validación cruzada. Los dos ejemplares de Quibas se asocian a E. a. altidens, si bien se observa que la dispersión de E. a. altidens es mucho mayor que la de E. a. granatensis. El astrágalo no es una pieza muy significativa desde el punto de vista taxonómico.

En el caso de los MtIII, los resultados indican una clasificación previa correcta del $78,9 \%$ de los casos, mientras que por validación cruzada es del 70,4\%. De los dos ejemplares de Quibas, uno queda clasificado como E. a. altidens y el otro como E. a. granatensis, como ocurre con los metacarpianos. En este caso observamos que los restos de Quibas parecen ser de todas maneras más próximos a E. a. altidens.

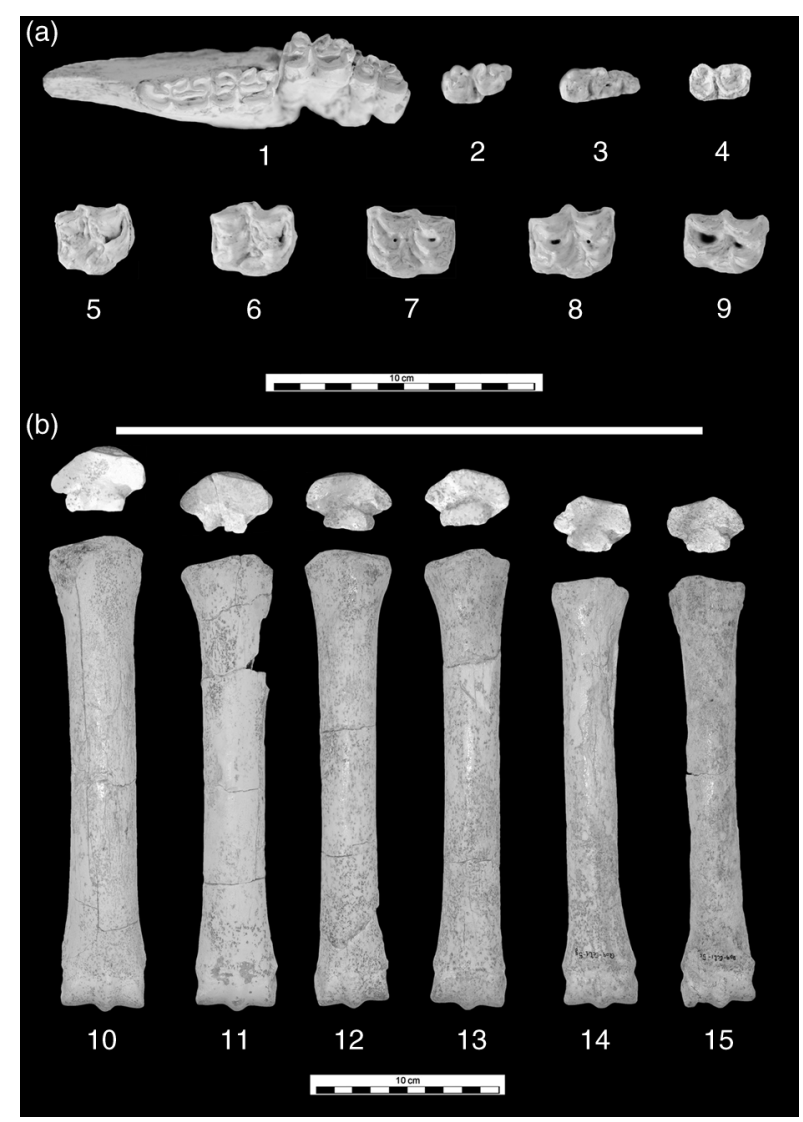

Fig. 3.-A, restos dentarios en vista oclusal procedentes de la localidad de Quibas (Murcia). Equus altidens: 1, hemimandíbula izquierda con la serie p2-m1 -Q03-2 2 -1(a)-; 2, m2 izquierdo -Q03$22_{2}-1$ (b), forma parte de la hemimandíbula (1)-; $3, \mathrm{~m} 3$ izquierdo -Q03-2 2 -1(b), forma parte de la hemimandíbula (1)-; 4, m1-2 izquierdo (Q03-E20); 5, P3-4 derecho (Q07-Ect-34g); 6, P3-4 derecho (Q07-Ect-34f); 7, M1-2 izquierdo (Q07-Ect-34a); 8, M1-2 derecho (Q07-Ect-34b). E. suessenbornensis: 9, M1-2 derecho en germen (Q07-Ect-34c). B, metacarpianos en vista proximal -arriba- y vista anterior -abajo-: 10, metacarpo III izquierdo (Q09-Ect1-10c); 11, metacarpo III izquierdo (Q07-Ect-150); 12 , metacarpo III derecho (Q08-Ect1-11a); 13, metacarpo III izquierdo (Q09-Ect1-21a); 14, metacarpo III izquierdo (Q09-Ect1-5g); 15, metacarpo III derecho (Q09-Ect1-5c).

En las 1FIII, el 90,9\% de los casos agrupados originalmente se clasifican correctamente, mientras que la clasificación es correcta en el 87,3\% mediante validación cruzada. Los cinco ejemplares de 1FIII procedentes del yacimiento de Quibas quedan agrupados en la subespecie E. a. altidens. Como en los casos previos, observamos que la dispersión de los restos de E. a. altidens es bastante mayor que la de los de E. a. granatensis.

Los resultados para las 2FIII indican una clasificación previa correcta del $77,8 \%$ de los casos y del 


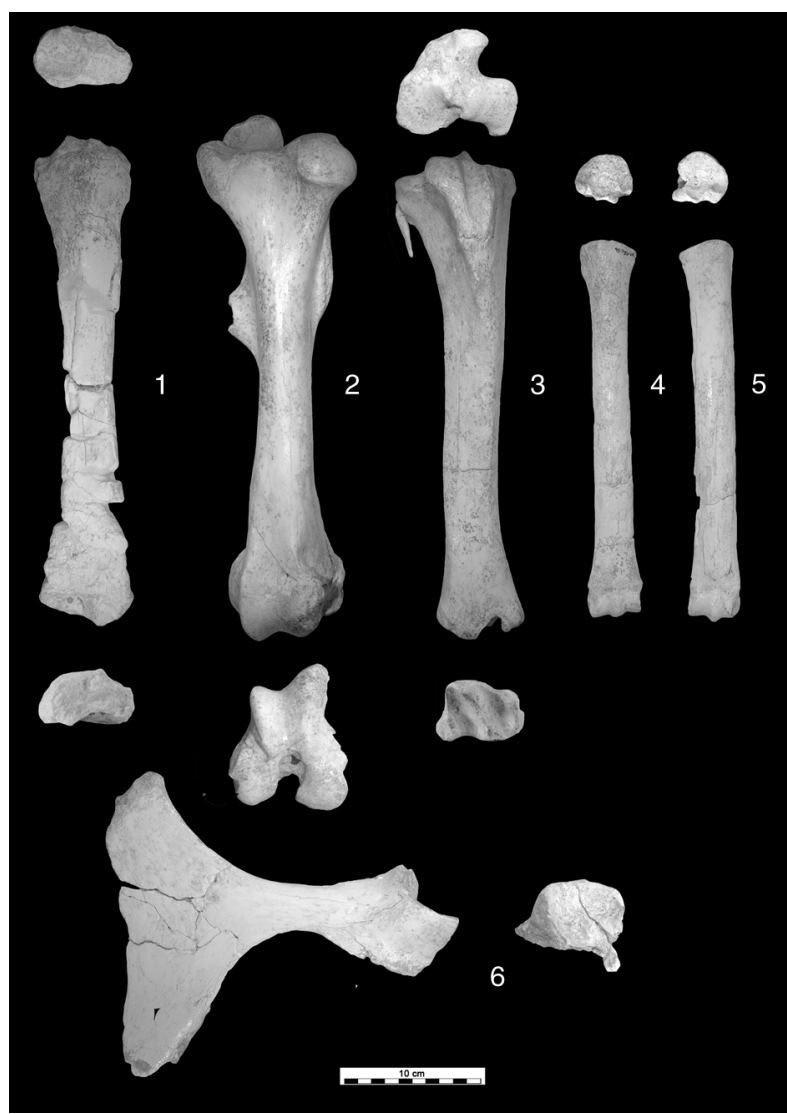

Fig. 4.-Huesos largos, metatarsianos y pelvis de Equus altidens procedentes de la localidad de Quibas (Murcia). 1, radio derecho en vista proximal -arriba-, en vista anterior -centro- y en vista distal -abajo- (Q07-Ect-149); 2, fémur derecho en vista anterior -arriba- y en vista distal -abajo- (Q08-Ect1-10a); 3, tibia derecha en vista proximal -arriba-, en vista anterior -centro- y en vista distal -abajo- (Q08-Ect1-10a); 4, metatarso III derecho en vista proximal -arriba- y en vista anterior -abajo- (Q09-Ect1-5b); 5, metatarso III izquierdo en vista proximal -arriba- y en vista anterior -abajo- (Q09-Ect1-12a); 6, pelvis en vista anterior -izquierda- y vista del acetábulo -derecha- (Q09-Ect1-12c).

$74,1 \%$ de los casos con validación cruzada. De las seis 2FIII de Quibas, cinco se agrupan con E. a. altidens, y una con E. a. granatensis. Tampoco las segundas falanges centrales son muy significativas desde el punto de vista taxonómico, siendo muy parecidas en los caballos de tamaño similar.

\section{Discusión y conclusiones}

Se ha podido comprobar, tanto con los caracteres morfológicos de los dientes y los huesos, como con los resultados de los análisis discriminantes, que al menos el 97\% de los restos de Quibas

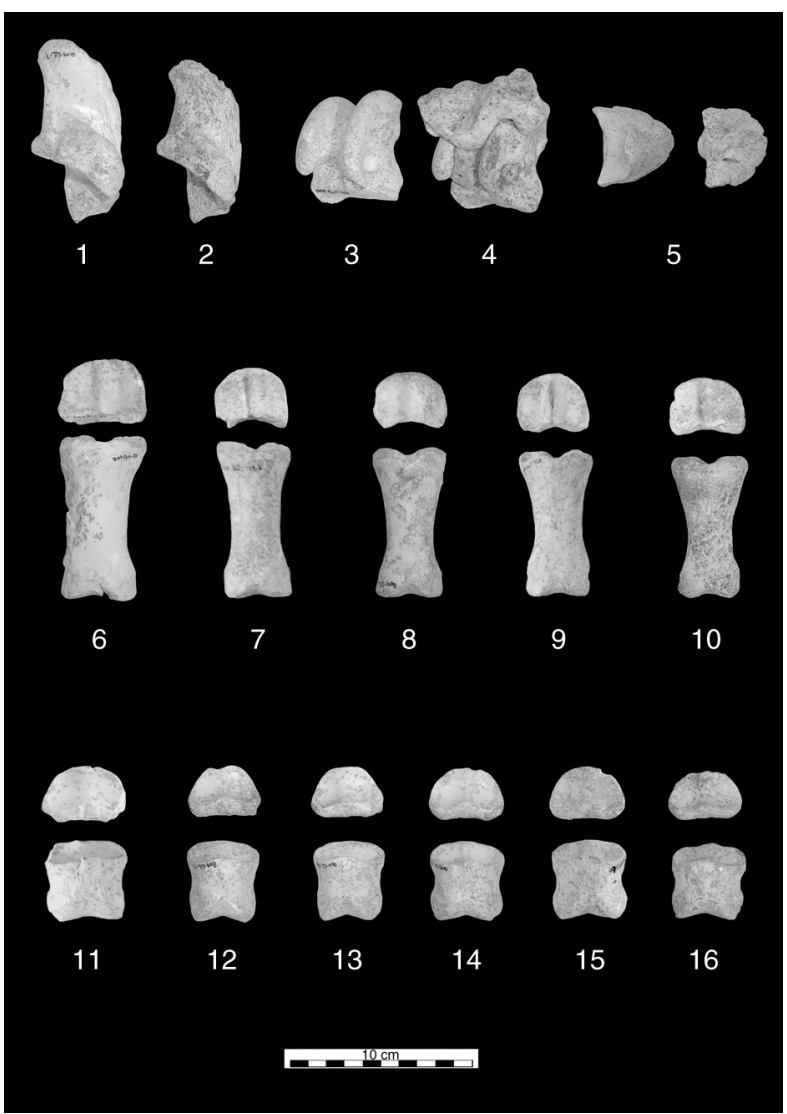

Fig. 5.-Calcáneos, astrágalos, y falanges procedentes de la localidad de Quibas (Murcia). 1, calcáneo derecho en vista medial (Q09-Ect1-7); 2, calcáneo derecho en vista medial (Q09-Ect1-2d); 3, astrágalo derecho en vista anterior (Q08-Ect1-10n); 4, astrágalo derecho en vista anterior (Q09-Ect1-1f); 5, $3^{\mathrm{a}}$ falange III en vista anterior -izquierda- y vista posterior -derecha- (Q09-Ect1-5h); 6, $1^{\mathrm{a}}$ falange III en vista proximal -arriba- $\mathrm{y}$ en vista anterior -abajo(Q09-Ect1-21b); 7, $1^{\text {a }}$ falange III en vista proximal -arriba- y en vista anterior -abajo- (Q08-Ect1-11b); 8, $1^{\mathrm{a}}$ falange III en vista proximal -arriba- y en vista anterior -abajo- (Q09-Ect1-5d); 9, $1^{\text {a }}$ falange III en vista proximal -arriba- y en vista anterior -abajo(Q08-Ect1-10d); 10, $1^{\text {a }}$ falange III en vista proximal -arriba- y en vista anterior -abajo- (Q09-Ect1-2i); 11, $2^{\mathrm{a}}$ falange III en vista proximal -arriba- y en vista anterior -abajo- (Q09-Ect1-3c); 12, $2^{\mathrm{a}}$ falange III en vista proximal -arriba- y en vista anterior -abajo(Q09-Ect1-10c); 13, $2^{\mathrm{a}}$ falange III en vista proximal -arriba- y en vista anterior -abajo- (Q09-Ect1-10e); 14, $2^{\mathrm{a}}$ falange III en vista proximal -arriba- y en vista anterior -abajo- (Q09-Ect1-3); 15, $2^{\mathrm{a}}$ falange III en vista proximal -arriba- y en vista anterior -abajo(Q08-Ect1-10g); 16, $2^{\mathrm{a}}$ falange III en vista proximal -arriba- $y$ en vista anterior -abajo- (Q09-Ect1-5e).

corresponde a un caballo de talla media de tipo estenonoide, aunque más grácil que los de las subespecies de E. stenonis y similar a las de E. altidens. Además, los análisis bivariantes permiten constatar la escasa presencia del caballo de mayor talla 

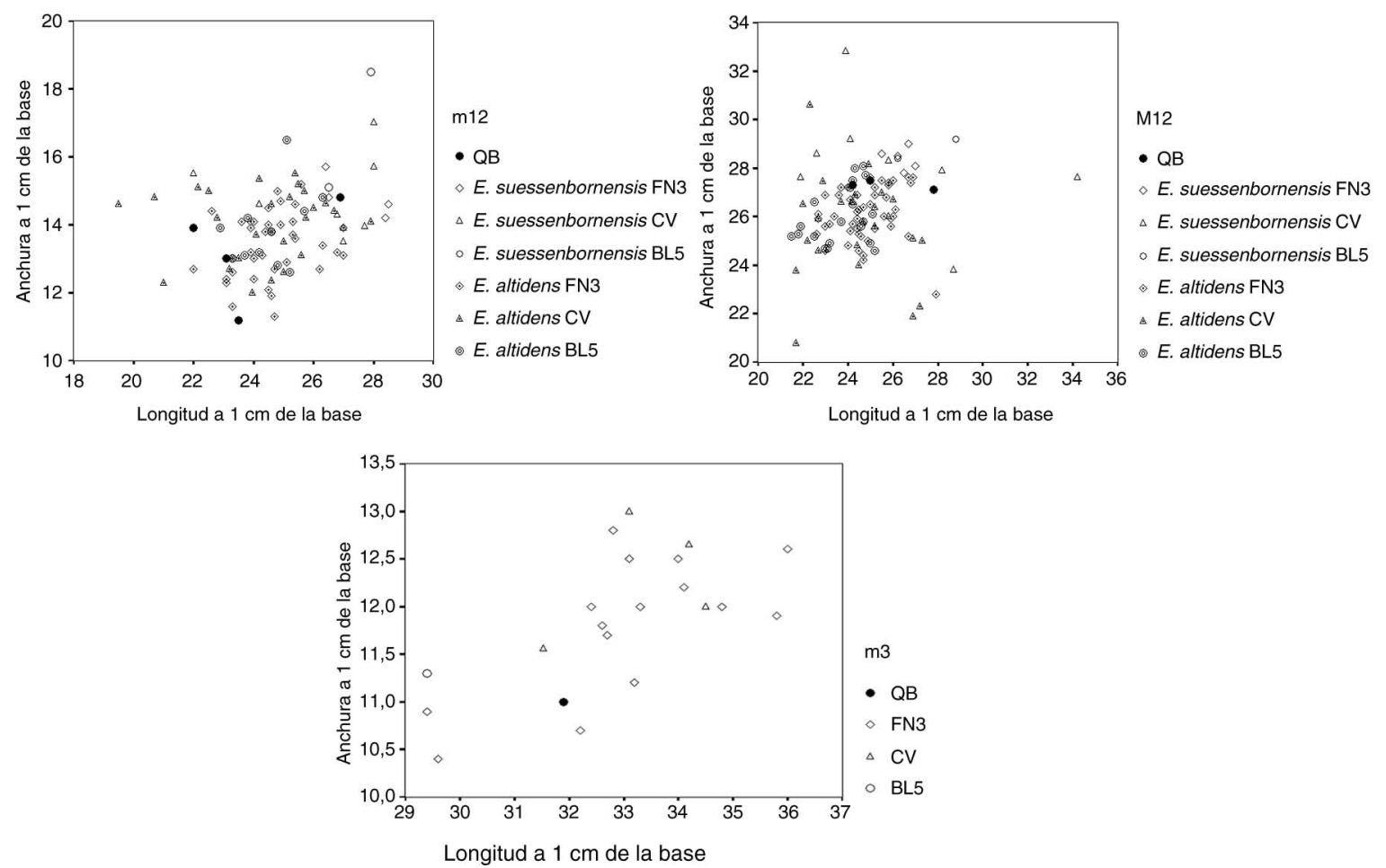

Fig. 6.-Diagrama bivariante de los dientes M1-2, m1-2 y m3 del yacimiento de Quibas (Murcia). Abreviaturas: QB, Quibas; FN3, Fuente Nueva 3; CV, Cueva Victoria; BL5, Barranco León 5.

Equus suessenbornensis. En los análisis estadísticos se observa un solapamiento entre las dos subespecies de E. altidens, correspondientes a los extremos de la distribución de ambas formas, siendo mayor en los casos del astrágalo, el calcáneo y las falanges. En el caso del McIII, donde tres de las cuatro piezas se agrupan con E. a. granatensis, se debe tener en cuenta que los restos de esta subespecie son más numerosos en el conjunto de la base de datos que disponemos, lo que trae consigo que esta forma tenga más peso en el análisis. Esto apoya la determinación preliminar realizada por Montoya et al. (1999) de que los restos de Quibas corresponden a E. altidens, último representante de los stenonoides europeos. Si algún día se dispone de un mayor número de fósiles en esta localidad podremos llegar a su determinación subespecífica.

Desde el punto de vista filogenético, no hay consenso sobre el origen estenoniano de E. altidens, el cual podría estar en Equus livenzovensis (primer stenonoide europeo, Plioceno Superior; localidad tipo Livenzovka, sur de Rusia) según Alberdi et al. (1998). Este planteamiento marcaría la evolución desde unos primeros caballos estenonianos, bastante pesados, hasta los más gráciles a los que corresponde esta forma de Quibas (Alberdi et al., 1998; Forsten, 1986). Sin embargo, otros autores consideran que E. altidens tendría su origen en una diferenciación a partir de Equus numidicus en África (Guerrero-Alba \& Palmqvist, 1997), mientras que Eisenmann (2004) lo incluye en un grupo no estenoniano de équidos gráciles dispersados ampliamente en el Pleistoceno Inferior y Medio. Según Alberdi et al. (1998) se trata del último representante de los estenonianos en Europa, opinión que compartimos aquí, ya que los rasgos de E. altidens están vinculados a la línea evolutiva de E. stenonis planteada por estos autores.

En cuanto a las relaciones filéticas de Equus suessenbornesis, hay mucha discusión. Así, hay autores que lo consideran muy cercano o directamente descendiente de E. major (Grossouvre \& Stehlin, 1912; Nobis, 1971; Samson, 1975; Azzaroli, 1984; Forsten, 1999; Alberdi et al., 1998, 2001). Otros lo consideran un caballo primitivo o un verdadero caballino (Gromova, 1949). Musil (1969, 1992) considera que no tienen ninguna relación con los estenonianos. 

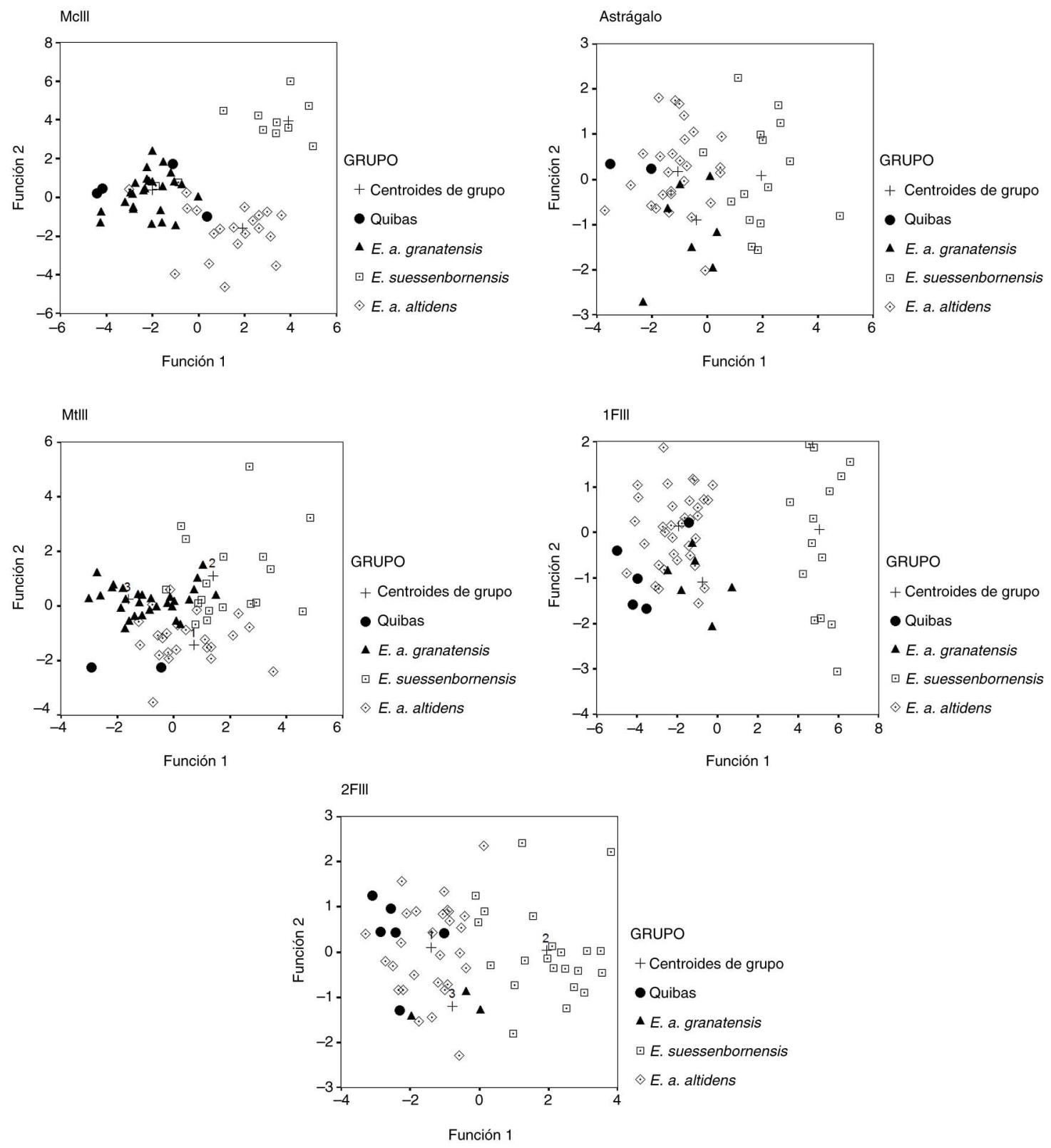

Fig. 7.-Diagramas de los análisis discriminantes de McIII, astrágalos, MtIII, 1FIII y 2FIII.

Por otra parte, los datos morfológicos y biométricos sugieren que esta especie podría pertenecer a la línea filética E. major - E. suessenbornensis, probablemente originada a partir del clado de E. livenzovensis (Alberdi et al., 1998).

La disminución del peso del cuerpo en los caballos estenonianos podría estar vinculada con los cambios climáticos y consecuentemente vegetacionales que se dieron desde mediados del Villafranquiense hasta el principio del Galeriense. Equus altidens se relaciona en España con unas condiciones climáticas cálidas o cálidas-templadas (Sánchez et al., 1994). Durante el período mencionado, el clima predominante en Europa occidental habría sido cálido y húmedo, cuya vegetación principal estaría compuesta de bosque o bosque-sabana (Bonifay, 1990; Guérin, 1984; Meón et al., 1980). El caballo de gran tamaño E. suessenbornensis, está escasamente representado en Quibas, 
al igual que en la mayoría de las localidades donde se presenta su asociación con E. altidens (Huéscar-1, Cúllar de Baza-1, Fuente Nueva-3 o Barranco León-5 en la cuenca de Guadix-Baza; Alberdi et al., 1998; Alberdi, 2010). Representan un caso de simpatría en el que la gran diferencia en su masa corporal sugiere que ambas especies podrían ocupar distintos nichos ligados a diferencias ecológicas que evitarían problemas de competencia (Forsten 1988; Alberdi et al., 1995, 1998).

\section{AGRADECIMIENTOS}

Queremos dar las gracias a M. Mancheño y G. Romero, quienes nos permitieron el estudio de este material. Al Museo Arqueológico de Murcia por habernos dado todas las facilidades posibles para el estudio del mismo. Igualmente damos las gracias al equipo de excavación que colaboró en el proceso de recuperación de los restos fósiles. Este trabajo ha sido posible en el marco de los Proyectos PI-676-04 (7507) de la Fundación Séneca (Murcia) y CGL2010-19116/BOS de la DGCYT. P. Piñero está sujeto a la Beca Predoctoral FPU (FPU12/02668) con la financiación del Ministerio de Educación Cultura y Deportes. Por último queríamos agradecer a E. Cerdeño su labor como revisora de este artículo por sus valiosos comentarios sobre el mismo.

\section{Referencias}

Alba, D.M.; Carlos-Calero, J.A.; Mancheño, M.A.; Montoya, P.; Morales, J. \& Rook, L. (2011). Fossil remains of Macaca sylvanus florentina (Cocchi, 1872) (Primates, Cercopithecidae) from the Early Pleistocene of Quibas (Murcia, Spain). Journal of Human Evolution, 61 (6): 703-718. http://dx.doi. org/10.1016/j.jhevol.2011.09.003

Alberdi, M.T. (1974). El género Hipparion en España. Nuevas formas de Castilla y Andalucía, revisión e historia evolutiva. Trabajos sobre Neógeno y Cuaternario, 11: 1-146.

Alberdi, M.T. (2010). Estudio de los caballos de los yacimientos de Fuente Nueva-3 y Barranco León-5 (Granada). In: Ocupaciones humanas en el Pleistoceno inferior y medio de la Cuenca de Guadix-Baza (Toro, I.; Martínez-Navarro, B. \& Agustí, J., Eds.). Junta de Andalucía, Consejería de Cultura, p. 291-306.

Alberdi, M.T. \& Palombo, M.R. (2013a). The Early Pleistocene Equidae from Pirro Nord (Apricena, Southern Italy). Palaeontographica Abteilung A, Palaeozoologie-Stratigraphie, 298 (1-6): 147-167. http://dx.doi.org/10.1127/pala/298/2013/147

Alberdi, M.T. \& Palombo, M.R. (2013b). The late Early to early Middle Pleistocene stenonoid horses from Italy. Quaternary International, 288: 25-44. http:// dx.doi.org/10.1016/j.quaint.2011.12.005
Alberdi, M.T. \& Piñero, P. (2015). Estudio de los caballos del yacimiento de Cueva Victoria, Pleistoceno inferior (Murcia). In: Paleontología y Geología de Cueva Victoria (Gibert, L. \& Ferràndez-Cañadell, E., Eds.). Museo Arqueológico Municipal de Cartagena. p. 325-358.

Alberdi, M.T. \& Ruiz Bustos, A. (1989). Taxonomía y Bioestratigrafía de Equidae (Mammalia, Perissodactyla) en la Cuenca de Guadix-Baza (Granada). Geología y Paleontología de la Cuenca de GuadixBaza. Trabajos sobre el Neogeno-Cuaternario, 11: 239-270.

Alberdi, M.T.; Ortiz-Jaureguizar, E. \& Prado, J.L. (1998). A quantitative review of European stenonoid horses. Journal of Paleontology, 72 (2): 371-387.

Alberdi, M.T.; Ortiz Jaureguizar, E. \& Prado, J.L. (2001). Comments on "Equus species as stratigraphic markers. Reality or wishful thinking?" and "A review of Equus stenonis Cocchi (Perissodactyla, Equidae) and related forms" by A. Forsten. Quaternary Science Review, 20 (10): 1149-1152. http://dx.doi.org/10.1016/S02773791(00)00154-2

Alberdi, M.T.; Prado, J.L. \& Ortiz Jaureguizar, J. (1995). Patterns of body size changes in fossil and living Equini (Perissodactyla). Biological Journal of the Linnean Society, 54 (4): 349-370. http://dx.doi. org/10.1111/j.1095-8312.1995.tb01042.x

Azzaroli, A. (1983). Quaternary mammals and the "EndVillafranchian" dispersal event-A turning point in the history of Eurasia. Palaeogeography, Palaeoclimatology, Palaeoecology, 44 (1-2): 27-47. http:// dx.doi.org/10.1016/0031-0182(83)90008-1

Azzaroli, A. (1984). On some vertebrates remains of middle Pleistocene age from the Upper Valdarno and Val di Chiana, Tuscany. Palaeontographia Italica, 73: 104-115.

Bernor, R.L.; Tobien, H. \& Woodburne, M.O. (1990). Patterns of Old World Hipparionine evolutionary diversification and biogeographic extension. In: European Neogene Mammal Chronology (Lindsay, E.H.; Fahlbusch, V. \& Mein, P., Eds.), Plenum Press, New York. p. 263-319.

Bonifay, M.F. (1990). Relations between paleoclimatology and Plio-Pleistocene biostratigraphy data in west European countries. In: European Neogene Mammal Chronology (Lindsay, E.; Fahlbusch, V. \& Mein, P., Eds.), Nato ASI Serie A, Life Sciences, 180: 475-485.

Blain, H.A.; Bailón, S.; Agustí, J.; Piñero-García, P.; Lozano-Fernández, I.; Laplana, C.; Sevilla, P.; LópezGarcía, J.M; Romero, G. \& Mancheño, M.A. (2014). Youngest agamid lizards from Western Europe (Sierra de Quibas, Spain, late Early Pleistocene). Acta Paleontologica Polonica, 59 (4): 873-878. http://dx.doi. org/10.4202/app.2012.0141

Carlos-Calero, J.A.; Mancheño, M.A.; Montoya, P. \& Ruiz Bustos, A. (2004). El yacimiento pleistoceno de la Sierra de Quibas (Abanilla, Murcia). In: Libro de 
Resúmenes de las XX Jornadas de la Sociedad Española de Paleontología (Calonge, A.; Gozalo, R.; López Carrillo, M.D. \& Pardo M.V., Eds.), Universitiy of Alcalá de Henares, Madrid, Spain. p. 40-41.

Carlos-Calero, J.A.; Montoya, P.; Mancheño, M.A. \& Morales, J. (2006a). Presencia de Vulpes praeglacialis en el yacimiento pleistoceno de la sierra de Quibas (Murcia, España). Estudios Geológicos, 62 (1): 395-400. http://dx.doi.org/10.3989/egeol.0662134

Carlos-Calero, J.A.; Van der Made, J.; Mancheño, M.A.; Montoya, P. \& Romero, G. (2006b). Capra alba Moyà-Solà, 1987 del Pleistoceno inferior de la Sierra de Quibas (Murcia, España). Estudios Geológicos, 62 (1): 571-578. http://dx.doi.org/10.3989/egeol. 0662160

Cuadros, I.A. (2010). Aproximación tafonómica de los micromamíferos del yacimiento paleontológico de Quibas: Evidencias de digestión, fracturas y procesos diagenéticos. Proyecto fin de Máster, Universidad Complutense de Madrid, Madrid, Spain, 55 pp.

Durán, J.J.; López-Martínez, J. \& Mancheño, M.A. (2004). Dos registros de espeleotemas pleistocenos de gran potencia en la Península Ibérica: primeros resultados isotópicos. Boletín Geológico y Minero, 115 (2): 265-270.

Eisenmann, V. (2004). Les Equidés (Mammalia, Perissodactyla) de Saint-Vallier (Drôme, France) et les Equidés Plio-Pleistocènes d'Europe. Geobios, 37 (S1): 279-305. http://dx.doi.org/10.1016/S0016-6995(04) 80019-6

Eisenmann, V.; Alberdi, M.T.; De Giuli, C. \& Staesche, U. (1988). Collected papers after the "New York International Hipparion Conference, 1981". In: Studying fossil Horses, Methodology 1 (Woodburne, M. \& Sondaar, P., Eds.), E.J. Brill, Leiden, 77 p.

Forsten, A. (1986). A review of the Süssenborn horses and the origin of Equus hydruntinus Regalia. Quartärpaläontologie, 6: 43-52.

Forsten, A. (1988). Middle Pleistocene replacement of stenonid horses by caballoid horses - Ecological implications. Palaeogeography, Palaeoclimatology, Palaeoecology, 65 (1-2): 23-33. http://dx.doi.org/ 10.1016/0031-0182(88)90109-5

Forsten, A. (1999). A review of Equus stenonis Cocchi (Perissodactyla, Equidae) and related forms. Quaternary Science Review, 18 (12): 1373-1408. http:// dx.doi.org/10.1016/S0277-3791(98)00073-0

Gromova, V.I. (1949). Istorija loshadej (roda Equus) v Starom Svete. Chast' 1. Obzor i opisanie form. Trudy paleontological Instutet, 17: 1-373.

Grossouvre, A. \& Stehlin, H.G. (1912). Les sables de Rosières, près Saint-Florent (Cher). Bulletin de la Société Géologique de France, 4: 194-212.

Guérin, C. (1984). Grands mammifères marqueurs de l'environnement et des climats du Villafranchien d'Europe occidentale. Methodologies et resultats. Paléobiologie continentale, 14 (2): 287-299.
Guerrero-Alba, S. \& Palmqvist, P. (1997). Estudio morfométrico del caballo de Venta Micena (Orce, Granada) y su comparación con los équidos modernos y del Plio-Pleistoceno en el viejo mundo. Paleontologia i Evoluciò, 30-31: 93-148.

Lindsay, E.H.; Opdyke, N.D. \& Johnson, N.M. (1980). Pliocene dispersal of horse Equus and late Cenozoic mammalian dispersal events. Nature, 287: 135-138. http://dx.doi.org/10.1038/287135a0

Marín, M. (1987). Equus stenonis granatensis en el Pleistoceno inferior de Venta Micena (Granada, España). Paleontologia i Evolució, Memòria Especial, 1: 255-282.

Méon, H.; Ballesio, R.; Guérin, C. \& Mein, P. (1980). Approche climatologique du Néogène supérieur (Tortonien à Pléistocène moyen ancien) d'après les faunes et les flores d'Europe occidentale. Mémoires du Museum national d'histoire naturelle. Série B, Botanique, 27: 182-195.

Montoya, P.; Alberdi, M.T.; Barbadillo, L.J.; Van der Made, J.; Morales, J.; Murelaga, X.; Peñalver, E.; Robles, R.; Ruiz Bustos, A.; Sánchez, A.; Sanchiz, B.; Soria, S. \& Szyndlar, Z. (2001). Une faune très diversifiée du Pléistocène inférieur de la Sierra de Quibas (provincia de Murcia, Espagne). Comptes Rendus de l'Académie des sciences de Paris, Sciences de la Terre et des planètes, 332: 387-393. http://dx.doi. org/10.1016/S1251-8050(01)01544-0

Montoya, P.; Alberdi, M.T.; Blázquez, A.M.; Barbadillo, L.J.; Fumanal, M.P.; Van der Made, J.; Marín, J.M.; Molina, A.; Morales, J.; Murelaga, X.; Peñalver, E.; Robles, F.; Ruiz Bustos, A.; Sánchez, A.; Sanchiz, B.; Soria, S. \& Szyndlar, Z. (1999). La fauna del pleistoceno Inferior de la Sierra de Quibas (Abanilla, Murcia). Estudios Geológicos, 55 (3-4): 127-161. http://dx.doi.org/10.3989/egeol.99553-4171

Musil, R. (1969). Die Equiden-Reste aus dem Pleistozän von Süssenborn bei Weimar. Paläontologische Abhandlungen Paläozoologie, 3: 617-666.

Musil, R. (1992). Die Pferde aus der oberpliozanen Spaltenfallung Schemfeld bei Eichstgtt. Mitteilungen der Bayerischen Staatssammlung für Paläontologie und Historische Geologie, 32: 115-162.

Nobis, G. (1971). Vom Wilsdpferd zum Hauspferd. Böhlau Verlag Köln Wien, 96 p.

Piñero, P. (2012). Los roedores del Pleistoceno inferior de la Gruta1 del Complejo kárstico de Quibas. Proyecto fin de Máster, Universitat Autònoma de Barcelona y Universitat de Barcelona, Barcelona, Spain, 100 p.

Piñero, P.; Agustí, J.; Blain, H.A.; Furió, M.; Laplana, C. (2015). Biochronological data for the Early Pleistocene site of Quibas (SE Spain) inferred from rodents assemblage. Geologica Acta, 13 (3): 229-241. http:// dx.doi.org/10.1344/GeologicaActa2015.13.3.5

Prat, F. (1980). Les Équidés Villafranchiens en France. Genre Equus. CNRS, Paris, 290 p. 
Reyment, R.A. (1991). Multidimensional Paleobiology. Pergamon Press. $416 \mathrm{p}$.

Rodríguez-Estrella, T.; Mancheño, M.A.; Romero, G. \& Hernández, J.M. (2004). Características geológicas de la Sierra de Quibas (Abanilla, Murcia). Su relación con un yacimiento paleontológico pleistoceno. Geogaceta, 35: 115-118.

Samson, P. (1975). Les Equides fossiles de Roumanie. Geologica Romana, 14: 165-352.

Sánchez Chillón, B.; Alberdi, M.T.; Leone, G.; Bonadonna, F.P.; Stenni, B. \& Longinelli, A. (1994). Oxygen isotopic composition of fossil equid tooth and bone phosphate: an archive of difficult interpretation. Palaeogeography, Palaeoclimatology, Palaeoe-cology, 107 (3-4): 317-328. http://dx.doi.org/10.1016/0031-0182(94)90103-1

Van der Made, J.; Carlos Calero, J.A. \& Mancheño, M.A. (2007). New material of the got Capra alba from the Lower Pleistocene of Quibas and Huéscar (Spain). Notes on sexual dimorphism, stratigraphic disatribution and systematic. Bolletino della Società Italiana de Paleontología, 47: 13-23. 